\title{
Potential personality traits that explain cyberbullying among youth in Malaysia
}

\begin{abstract}
This study aims to produce a comprehensive understanding on cyberbullying among youth from the perspective of personality traits. The conceptual understanding on cyberbullying was gained by analysing available documents and literature. Several personality traits namely loneliness, self-esteem and empathy were found to influence cyberbullying among youth. Additionally, cyber victim experience was found to be another influential factor for cyberbullying. This article attempts to provide a way forward and highlight the need for concerned parties to understand cyberbullying among youth in Malaysia.
\end{abstract}

Keyword: Personality traits; Youth; Cyberbullying 